Revue des patrimoines

$40 \mid 2019$

Lits historiques. Première anthologie des lits européens du XVe au XIXe siècle

\title{
Nascere, amare, morire. I letti (e i loro abitanti) nella pittura bolognese tra XVI e XVII secolo
}

\section{Michele Danieli}

\section{(2) OpenEdition}

\section{Journals}

Edizione digitale

URL: http://journals.openedition.org/insitu/22905

DOI: 10.4000/insitu.22905

ISSN: 1630-7305

Editore

Ministère de la Culture

Notizia bibliografica digitale

Michele Danieli, « Nascere, amare, morire. I letti (e i loro abitanti) nella pittura bolognese tra XVI e XVII secolo », In Situ [En ligne], 40 | 2019, mis en ligne le 23 septembre 2019, consulté le 04 octobre 2019. URL : http://journals.openedition.org/insitu/22905 ; DOI : 10.4000/insitu.22905

Questo documento è stato generato automaticamente il 4 octobre 2019.

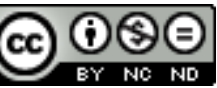

In Situ Revues des patrimoines est mis à disposition selon les termes de la licence Creative Commons Attribution - Pas d'Utilisation Commerciale - Pas de Modification 4.0 International. 


\title{
Nascere, amare, morire. I letti (e i loro abitanti) nella pittura bolognese tra XVI e XVII secolo
}

\author{
Michele Danieli
}

ie ne voyois point qu'on m'estimast inferieur a mes condisciples,

bien qu'il en eust desia entre eux quelques uns, qu'on destinoit a remplir les places des nos

maistres

R.D., 1637

1 Gli studi recenti, e gli interventi riuniti in questo volume, hanno messo in evidenza i diversi significati che si intrecciano a proposito del letto, che non è solo una parte dell'arredamento della dimora dell'età moderna, ma uno spazio al tempo stesso fisico e simbolico. ${ }^{1}$ La doppia natura dell'oggetto trova perfetta applicazione nella pittura del XVI secolo: qui infatti il letto si trasforma in una scenografia non meno che in un palcoscenico, sul quale si svolgono drammi e tragedie, vicende eroiche e amorose.

Il piccolo gruppo di dipinti che vorrei presentare non comprende che opere prodotte a Bologna tra la fine del XVI e l'inizio del XVII secolo. Si tratta quindi di una selezione molto circoscritta nel tempo e nello spazio (benché potenzialmente rappresenti l'embrione di una ricerca più vasta e sistematica), ma che proprio perché rinchiusa in confini così stretti illustra bene le diverse forme e significati dei letti qui illustrati $\mathrm{e}$ ritratti.

Due premesse sono necessarie. Non abbiamo preso in considerazione dipinti che illustrano l'Annunciazione, la Nascita della Vergine o la Nascita del Battista, in quanto in essi l'arredamento risponde quasi sempre a generiche esigenze iconografiche di sobrietà che conferiscono agli interni domestici un aspetto anonimo e stereotipato, poco utile ai fini del nostro discorso. ${ }^{2}$ In secondo luogo, bisogna ricordare che a Bologna non esiste un luogo plasticamente deputato all'esercizio del potere. Fin dalla fine del XIII secolo la città era sottoposta all'autorità papale; dal 1466 Giovanni II Bentivoglio tentò di 
imporre la propria signoria, ma nel 1506 (in seguito alle pressioni di papa Giulio II) la sua famiglia venne cacciata e la popolazione rase al suolo il suo palazzo, la "domus magna" centro della sua committenza e l'edificio più simile a una reggia rinascimentale che Bologna avesse mai conosciuto. Da allora, e fino alle campagne napoleoniche del 1796, Bologna rimase all'interno dello Stato Pontificio, governata da un Cardinale Legato nominato da Roma e rinnovato ogni cinque anni; alle famiglie nobili non rimaneva che spartirsi i quaranta seggi del Senato, aumentati a cinquanta nel 1589 proprio per mantenere una situazione di equilibrio e soffocare ogni tentazione di egemonia. Tutto questo per dire che Bologna, nonostante fosse un centro di produzione culturale e artistica di primaria importanza, non conobbe mai lo sfarzo di una civiltà di corte, né vi si produssero mai oggetti destinati a rappresentare il prestigio politicoculturale di un sovrano: né corone né residenze né, naturalmente, letti reali.

4 Il primo dipinto che vorrei considerare rappresenta Mercurio ordina a Enea di abbandonare Didone, eseguito da Orazio Samacchini intorno al 1570 e conservato al Museo del Louvre (fig. 1). ${ }^{3}$ Il testo dell' Eneide racconta la scena in una maniera completamente diversa: appena giunto in volo a Cartagine, Mercurio scorge Enea intento a tracciare strade e fortificazioni, con una spada ornata di diaspro alla cintura e un mantello di porpora sulle spalle. ${ }^{4} \mathrm{Al}$ contrario, Samacchini ambienta l'episodio in una camera, oltre la porta della quale si scorge una grande loggia; Enea, aiutato da un putto, si sta spogliando per raggiungere Didone quando Mercurio appare all'improvviso e gli ordina di abbandonare l'Africa.

Figure 1

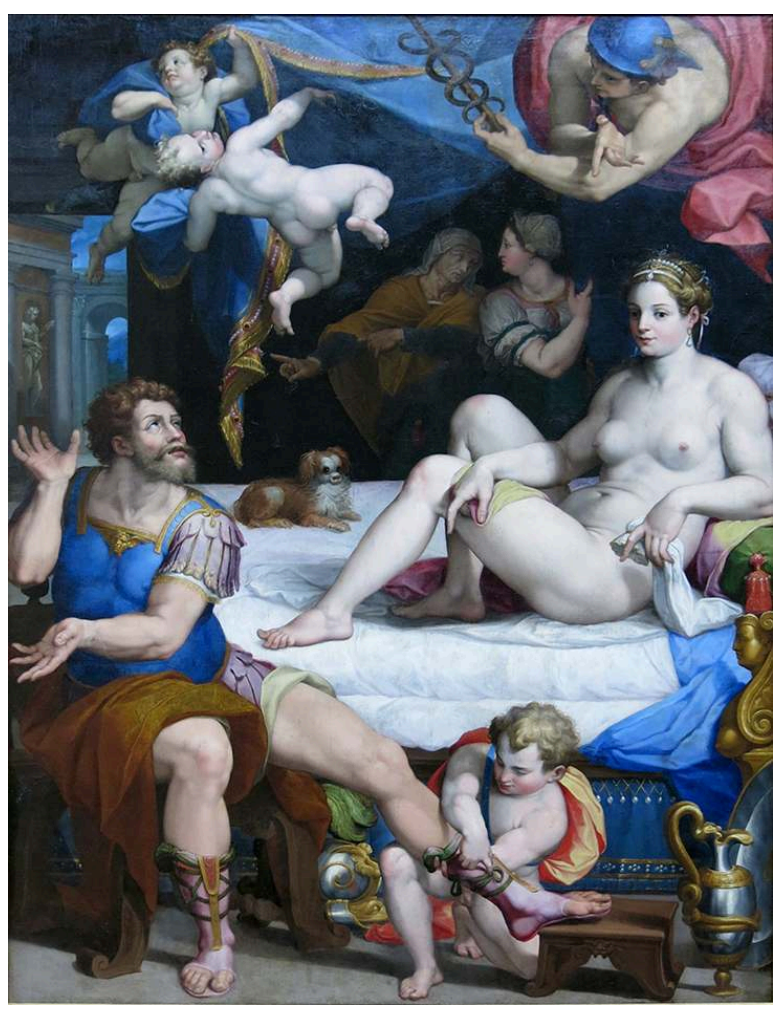

Orazio Samacchini, Mercure ordonne à Énée d'abandonner Didon. Paris, musée du Louvre. Phot. RMN-Louvre. (c) RMN-Louvre. 
Questa profonda differenza iconografica deriva sicuramente da un dipinto eseguito da Daniele da Volterra per Giovanni della Casa, descritto da Vasari: "Enea che, spogliandosi per andare a dormire con Dido, è sopragiunto da Mercurio". Il dipinto di Daniele oggi è perduto, ma la sua composizione è nota attraverso una copia e alcuni disegni, ed è piuttosto simile a quella di Samacchini. ${ }^{5} \mathrm{Nel}$ dipinto del Louvre, Didone ci appare seduta su un letto lussuoso, degno di una regina. Eppure questo letto è quasi completamente nascosto, tanto che non sapremmo descriverne la struttura. Più che illustrato, lo sfarzo è suggerito dal doppio materasso, dalle frange dorate del sopracielo (o forse sono cortine?) sollevato dai putti e dai ricchi ricami del tornaletto.

6 Nell'immagine creata da Samacchini si fondono diverse iconografie: oltre all'episodio virgiliano (molto raro nel XVI secolo) mi sembra che il gesto del putto che armeggia con il calzare di Enea riprenda un altro tema nuziale, ovvero l'episodio di Alessandro e Rossane. Come è noto, la fortuna iconografica del soggetto è legata alla descrizione che Luciano di Samosata dedicò all'opera del pittore Aezione, dove il particolare del putto che sfila il sandalo a Rossane è esplicitamente ricordato. ${ }^{6}$ Qui i ruoli sono invertiti, e non è nemmeno ben chiaro se Enea si stia vestendo o svestendo; credo tuttavia che il putto svolga la stessa funzione di quelli che, nella descrizione di Luciano, giocavano con le armi di Enea: "il pittore vuole mostrare l'amore che Alessandro nutriva anche per la guerra e indicare che, pur nel suo amore per Rossane, egli non poteva dimenticare le armi".

7 Subito dopo i due protagonisti principali, il personaggio più importante è proprio il letto, che occupa quasi tutto lo spazio della camera estendendosi in profondità. Oltre che un talamo nuziale, le sue dimensioni imponenti lo rendono anche un letto di rappresentanza dove la regina trascorre la sua giornata, acconciata con grande cura, assistita dalle ancelle e in compagnia di un cagnolino che non può essere, come di consueto, simbolo di fedeltà: sia Enea che Didone sono in qualche modo adulteri, in quanto il loro amore tradisce la memoria dei rispettivi coniugi defunti (e in questo dissidio interiore risiede gran parte del dramma). La dimensione in qualche modo "pubblica" del letto accentua il carattere erotico della nudità di Didone, tanto evidente da attirare la censura dei restauratori ottocenteschi, che ne avevano occultato il corpo sotto un improbabile panneggio ocra. ${ }^{7}$

Nel Suicidio di Lucrezia di Denys Calvaert compare invece un letto del tutto diverso (fig. 2). L'opera del pittore di Anversa (ma che trascorse quasi tutta la sua carriera a Bologna) si conserva al Musée Magnin di Digione e appartiene alla sua fase tarda, e forse si deve collocare già nei primi anni del Seicento, quando l'artista si dedicò con maggiore frequenza alla raffigurazione di grandi figure femminili nude. ${ }^{8}$ 
Figure 2

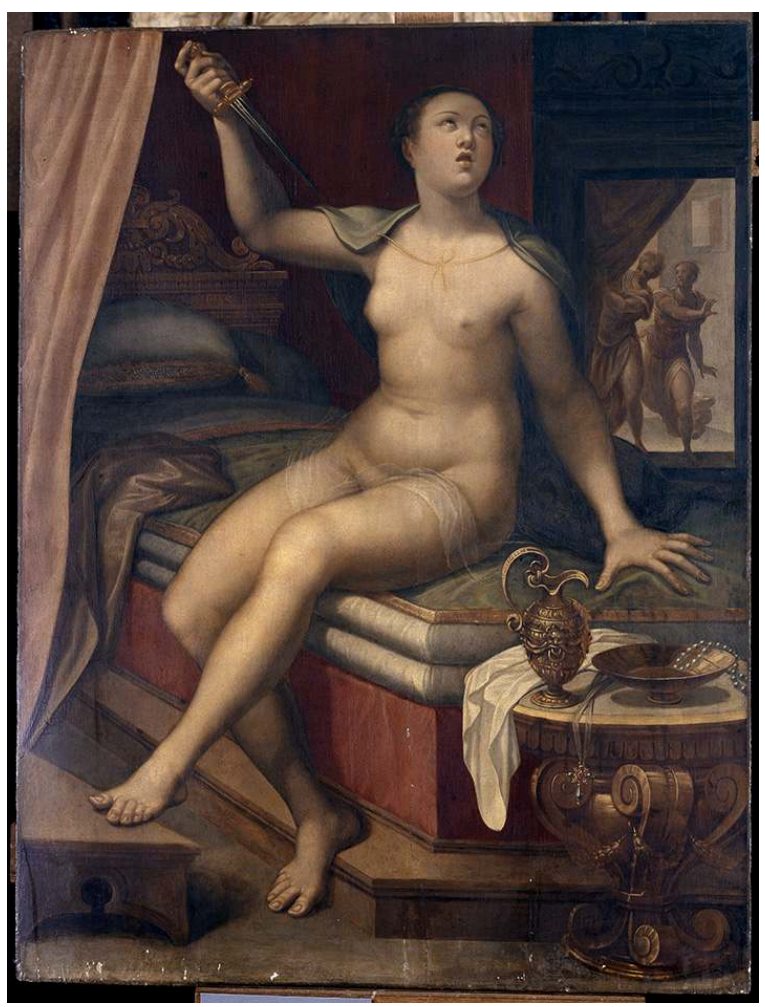

Denis Calvaert, Suicide de Lucrèce. Dijon, musée Magnin.

Phot. Franck Raux. (c) RMN-Grand Palais (musée Magnin).

9 Rispetto a quello di Samacchini, il letto raffigurato da Calvaert è molto più piccolo, forse persino leggermente sproporzionato poiché l'artista ha voluto conferire maggiore monumentalità alla figura. Inoltre, cosa più importante, ha un aspetto raccolto che evoca una dimensione assolutamente privata: le cortine infatti sono appena scostate, per permetterci di osservare la scena.

Il dipinto è noto da diverso tempo, ma soltanto osservandolo nel contesto di questa giornata di studi possiamo renderci conto della scelta compiuta dal pittore, che fa sedere Lucrezia su un letto decisamente démodé. Una coperta verde con una semplice bordatura copre due materassi piuttosto sottili; le cortine e il tornaletto rossi non presentano alcuna decorazione. Unico elemento di una certa rilevanza è la testiera decorata da un motivo a girali vegetali. Si tratta quindi di un mobile tutto sommato modesto, meno ricercato ad esempio della brocca cesellata e del tavolino posto in primo piano. Intorno alla base del letto corre un gradino, che sembra un ricordo delle cassapanche che circondavano i letti quattrocenteschi: si pensi soltanto al celebre letto Davanzati del Metropolitan Museum (nonostante i dubbi sulla sua effettiva datazione).

Si ha la sensazione che l'artista abbia scelto di raffigurare un letto dalla foggia antiquata non tanto per ambientare l'episodio in un tempo remoto ormai lontanissimo e irraggiungibile (la Roma della fine del VI secolo a.C.), ma per sottolineare la fermezza e il rigore morale della casta Lucrezia. Del resto Calvaert dimostra di conoscere bene la moda contemporanea in altri dipinti coevi: è più moderno e raffinato, ad esempio, il letto sul quale decide di togliersi la vita la più lussuriosa Cleopatra, in un dipinto di collezione privata. Anche il letto che compare in un bel rame con Giove e Semele è ricco e decorato (fig. 3): e si noti che le lenzuola sono in disordine poiché l'episodio fatale, 
secondo il racconto di Ovidio, si svolge durante un convegno amoroso tra la fanciulla e il dio. Al contrario il letto di Lucrezia è intatto, nonostante vi si fosse consumata la violenza sessuale. ${ }^{9}$

\section{Figure 3}

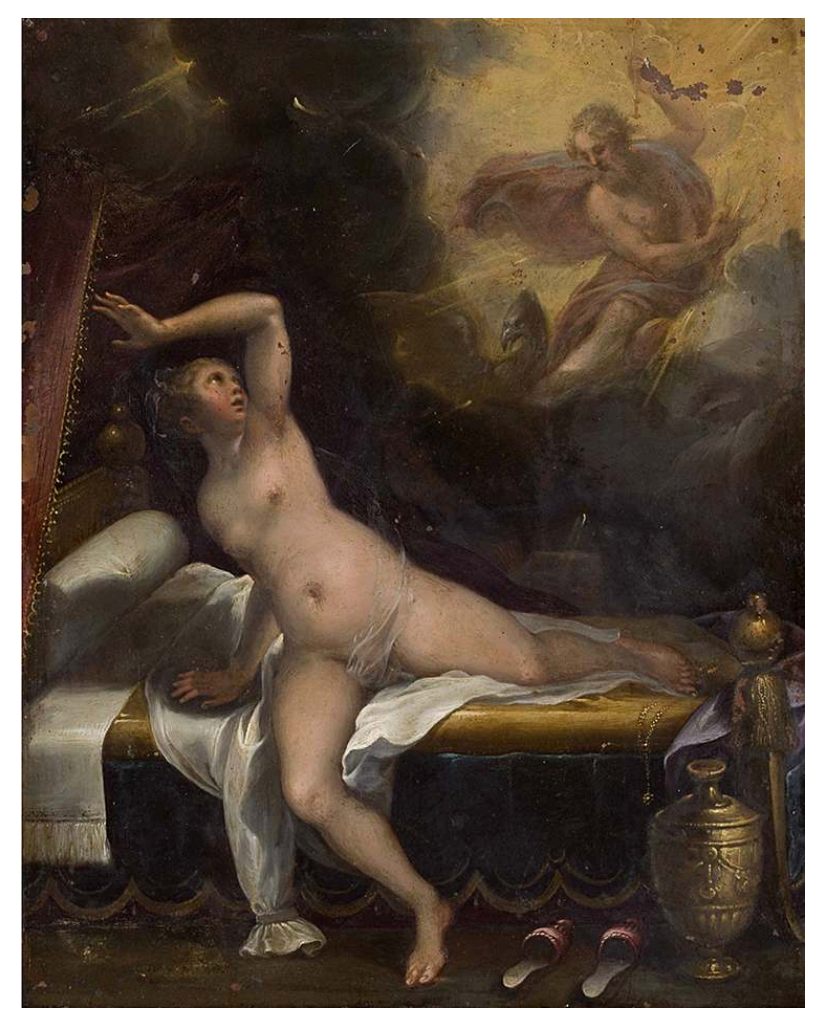

Denis Calvaert, Jupiter et Sémélé.

Phot. Michele Danieli. Collection particulière.

L'atteggiamento dell'artista, che utilizza anche l'arredamento per dare alla scena la giusta intonazione narrativa, deve metterci in guardia riguardo la precisione delle fonti visive nella descrizione degli oggetti contemporanei, e dalla tentazione di considerarli come moderni ready-made capitati quasi per caso all'interno dell'inquadratura.

Il letto che compare in un dipinto di Lorenzo Sabatini conservato alla Galleria Sabauda di Torino non svolge vere e proprie funzioni di arredamento, ma serve a evocare simbolicamente un contesto matrimoniale, e diventa fondamentale attributo iconografico di una raffigurazione allegorica (fig. 4). Il quadro fu acquistato intorno al 1725 dal principe Eugenio di Savoia come opera di Pellegrino Tibaldi, e fu esposto nella galleria della sua residenza viennese, oggi nota come il Belvedere. In un inventario del 1736 la sua appartenenza alla scuola bolognese era già stata dimenticata, ed era attribuito al fiorentino Francesco Salviati: "Une femme tenante dans une main un miroir et dans l'autre un compas, qui répresent la géometrie, de Salviati". Per quanto sbagliata, l'attribuzione a Salviati sopravvisse fino ad anni recenti, prima di venire corretta in favore di Sabatini. ${ }^{10}$ 
Figure 4

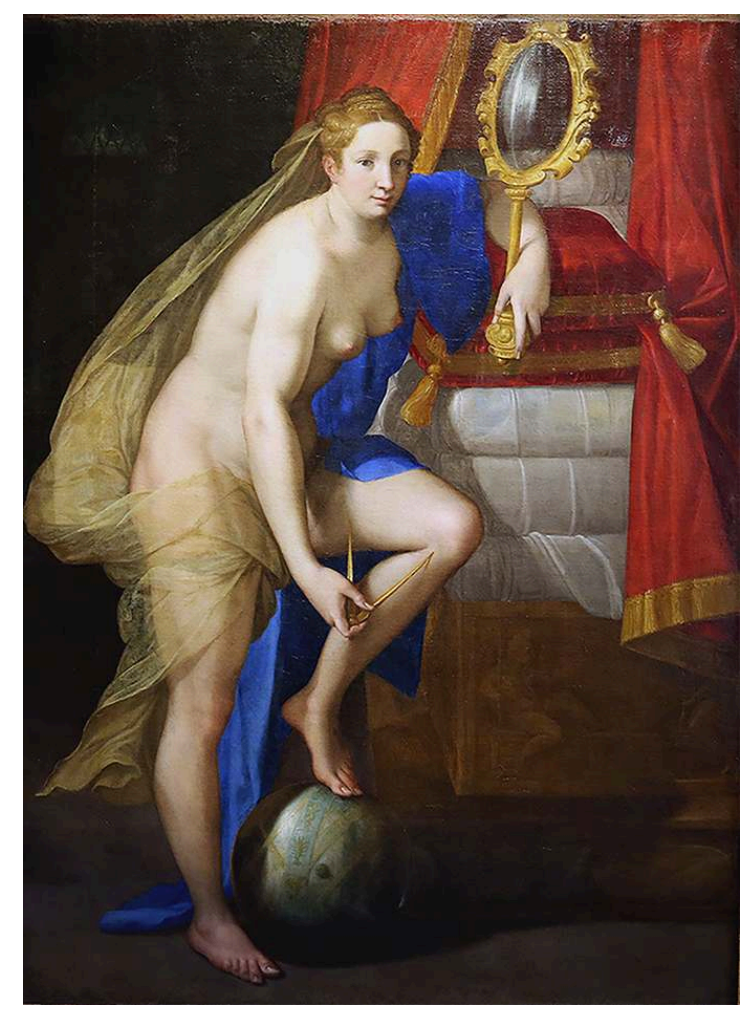

Lorenzo Sabatini, Le «mariage parfait ». Turin, Galleria Sabauda.

Phot. Michele Danieli. @ Galleria Sabauda, Turin.

14 L'interpretazione del soggetto è stata ancora più longeva: era indicato come Geometria già al momento della vendita a Eugenio di Savoia, e così è ancora oggi nel cartellino della Galleria Sabauda. Nell'Iconologia di Cesare Ripa (1593), la Geometria è descritta come "donna, che tenga in mano un Perpendicolo, \& con l'altra un Compasso", oppure "Donna, che con la destra tiene un Compasso, \& con la sinistra un Triangolo": come si vede, non vi è nessun accenno allo specchio, che nel nostro dipinto occupa una posizione troppo importante per potere essere trascurato. Non si possono accogliere nemmeno le ipotesi alternative di Mortari (1992), che propone di identificare la figura con la Simmetria, e di Benati che la vorrebbe riconoscere con una "Giustizia che si mira nello specchio" citata da un manoscritto settecentesco in un palazzo bolognese. ${ }^{11}$

15 Nessuno tuttavia si è mai chiesto il motivo per cui, alle spalle di una figura allegorica che dovrebbe in qualche modo essere connessa alla matematica e alle scienze geometriche, comparisse un grande letto. È evidente che non si tratta di un dettaglio compositivo trascurabile, poiché è posto in grande evidenza e descritto in ogni particolare. Per sciogliere il dubbio dobbiamo riconsiderare la figura femminile e i suoi attributi. Rivolgendoci ancora al testo di Ripa incontriamo la descrizione della Operazione perfetta: "donna, che tiene con la destra mano uno Specchio, \& con la sinistra un Squadro, \& un Compasso". ${ }^{12}$ L'incisione della Operazione perfetta nella prima edizione illustrata della Iconologia (1603) è molto vicina al dipinto di Sabatini.

16 Sembra dunque plausibile immaginare che il quadro della Galleria Sabauda sia stato dipinto in occasione di un matrimonio, e che rappresenti l'augurio di una perfetta unione coniugale. E per trasportare il significato dell'Operazione perfetta in ambito matrimoniale, cosa c'è meglio di un letto? Nel nostro caso, nonostante sia posto in 
grande evidenza, si tratta di un letto soltanto evocato del quale non vediamo che una piccola porzione: un talamo lussuoso, altissimo, fornito addirittura di tre materassi, allusione a piaceri coniugali confortevoli. Del resto non si spiegherebbero altrimenti alcuni particolari non secondari, come gli amorini raffigurati a bassorilievo sul basamento, e soprattutto la nudità della donna, cui il testo di Ripa non fa alcun accenno.

Nel nostro ultimo dipinto, il ruolo del letto è completamente diverso dai precedenti. Si tratta del Ritratto di neonato di Lavinia Fontana, conservato alla Pinacoteca Nazionale di Bologna (fig. 5), che motivi stilistici è stato datato intorno al 1583, e quindi è pressappoco contemporaneo ai dipinti che abbiamo visto finora. Nonostante Lavinia sia una grande specialista del genere, questo ritratto è isolato nella sua produzione e nonostante non si sia mai allontanato da Bologna è stato ricondotto alla pittrice soltanto nella seconda metà del XX secolo.

Figure 5

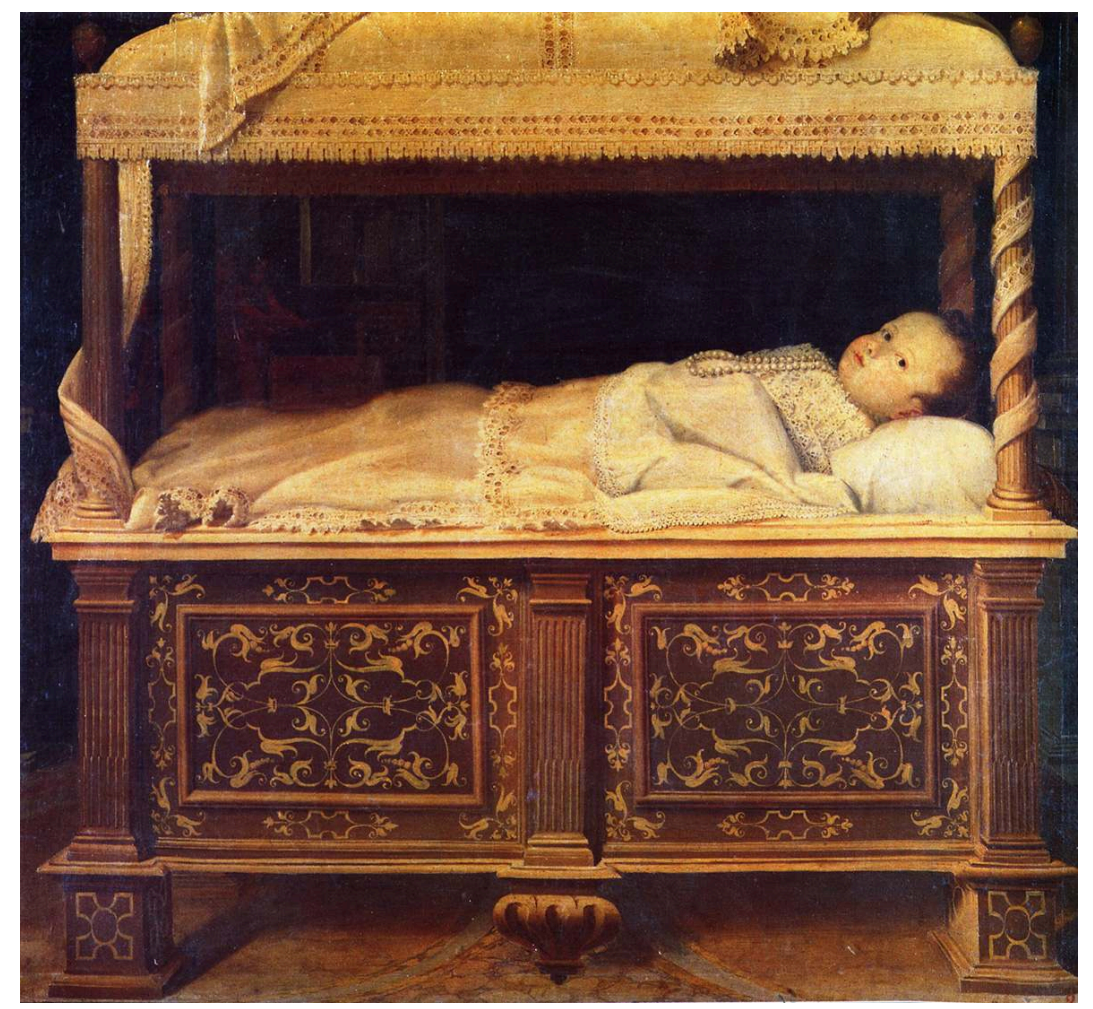

Lavinia Fontana, Portrait d'enfant. Bologne, Pinacothèque nationale.

(c) MIBACT - Archivio fotografico Pinacoteca Nazionale, Bologna.

Fu donato al Comune di Bologna nel 1761 dal conte Giulio Bianchetti, insieme a un altro dipinto raffigurante una dama della sua famiglia, la poetessa Giovanna Bianchetti vissuta nel XIV secolo: probabilmente anche il bambino apparteneva alla stessa famiglia, ma non ne conosciamo il nome. Al momento della donazione era accompagnato da una incredibile attribuzione a Leonardo che sopravvisse fino alla fine del XIX secolo; successivamente nei cataloghi della Pinacoteca si fecero i nomi di Federico Barocci e di Santi di Tito. ${ }^{13}$

19 In realtà già nel 1841, in una nota alla Felsina pittrice di Malvasia, compariva la soluzione del problema: "un quadro attribuito alla Lavinia rappresentante un Puttino reale che 
giace in un letticciuolo ornatissimo, involto in pannicelli e fregiato di collana" ${ }^{14}$ La ricchezza della culla e delle stoffe aveva fatto pensare a un Royal baby che però a Bologna (città senza una corte, come abbiamo detto in apertura) non sarebbe potuto nascere. Bisogna tuttavia riconoscere che nemmeno neonati più nobili, come il Federico della Rovere ritratto da Federico Barocci (1605), o il Leopoldo de' Medici di Tiberio Titi (1617), entrambi a Palazzo Pitti, avranno a loro disposizione culle così monumentali.

A differenza di quanto accade nel quadri di Samacchini, Calvaert e Sabatini, l'intenzione di Lavinia è quella di dipingere un mobile vero, testimonianza di un lusso solido, fatto di materiali durevoli e di artigianato sapiente, senza traccia di rêverie erotiche o allegoriche, sculture complicate o decorazioni dorate. La culla consiste in una struttura compatta, con tre lesene scanalate dai semplici capitelli che inquadrano due pannelli con intarsi vegetali. Quattro colonnine reggono un piccolo cielo ricoperto da una stoffa ricamata; nella parte alta corre una striscia di pendagli esterni.

La preziosità dell'insieme è esibita nel corredo di lenzuola ricamate, che costituivano una parte importante della ricchezza domestica, oggi quasi completamente perduta e dimenticata. Il nostro quadro vuole comunicare una condizione che si basa su una ricchezza solida, ben radicata nelle convenzioni del tempo, espressione di una piccola nobiltà ben attenta a sottolineare tutti gli elementi di distinzione sociale che era possibile inserire in questo contesto: la collana di perle, una dimora tanto spaziosa che è possibile destinare una intera stanza soltanto al piccolo letto, e naturalmente un corredo di prima qualità. $\mathrm{E}$ anche il ritratto di un bambino, certamente, ma soprattutto il ritratto di un letto bolognese della fine del Cinquecento.

\section{NOTE}

1. - AJMAR-WOLLHEIM Marta, DENNIS Flora (éd.), At Home in Renaissance Italy. London : V \& A Publications, 2006; CAVALLO Sandra, STOREY Tessa, Healthy Living in Late Renaissance Italy. Oxford : Oxford University Press, 2013, pp. 113-144 (“A Good Night's Sleep”); si veda anche la casistica riuntita da LEZOTTE Annette, The Home Setting in Early Netherlandish Paintings: A Statistical and Iconographical Analysis of Fifteenth- and Early Sixteenth-century Domestic Imagery. Lewiston : Mellen Press, 2008; si può citare anche un libro divulgativo come FRUGONI Chiara, Vivere nel Medioevo. Donne, uomini e soprattutto bambini. Bologna : Il Mulino, 2017, pp. 13-38 ("Felicità domestica, il letto e i suoi usi").

2. - Non fa eccezione nemmeno la celebre Annunciazione di Ludovico Carracci della Pinacoteca Nazionale di Bologna (BROGI, Alessandro, Ludovico Carracci (1555-1619). Ozzano Emilia : Tipoarte, 2001, pp. 221-223).

3. - Inv. 4637, olio su tela, cm 249x178. Entrò nel 1797 con i sequestri della Rivoluzione, attribuito a Martin Fréminet; BREJON DE LAVERGNÉE, Arnauld, THIÉBAUT, Dominique. Catalogue sommaire illustré des peintures du musée du Louvre. Italie, Espagne, Allemagne, Grande-Bretagne et divers. Paris : RMN, 1979, p.234; WINKELMANN, Jürgen. «Orazio Samacchini». Dans FORTUNATI PIETRANTONIO. Pittura bolognese del '500. Casalecchio di Reno : Grafis, 1986, p. 636 ("fondamentale per il percorso di Orazio Samacchini”); HABERT, Jean, LOIRE, Stéphane, SCAILLIÉREZ, Cécile, THIÉBAUT, Dominique. Catalogue des peintures italiennes du Musée du Louvre. Catalogue sommaire. 
Paris : Gallimard, 2007, p. 99; http://www2.culture.gouv.fr/public/mistral/joconde_fr? ACTION=CHERCHER\&FIELD_1=REF\&VALUE_1=000PE027031.

4. - "Ut primum alatis tegeti magalia plantis, / Aeneam fundantem arces ac tecta novantem / conspicit: atque illi stellatua iaspide fulva / ensis erat tyrioque ardebat murice laena, / demissa ex umeris, dive quae munera Dido / fecerat et tenui telas discreverat auro" (VIRGILIO, Eneide, IV, 259-264).

5. - VASARI, Giorgio, Le vite de' più eccellenti pittori, scultori, e architettori. Firenze : Giunti, 1568, V, p. 545; la copia fu pubblicata da VOSS, Hermann, «Ein wiedergefundenes Bild des Daniele da Volterra ». Kunstchronik, 1922-1923, XXIV, pp.375-37; vedi anche CIARDI, Roberto Paolo, MORESCHINI, Benedetta, Daniele Ricciarelli. Da Volterra a Roma. Milano : Motta, 2004, pp. 232-240; HOCHMANN, Michel. "Les dessins et les peintures de Fulvio Orsini et la collection Farnèse ". Mélanges de l'École Française de Rome. Italie et Méditerranée, 1993, CV, 1, p. 76, n. 12; PAGLIANO, Eric, L'atelier de l'œuvre. Dessins italiens du Musée Fabre. Kortrijk : Snoeck, 2013, pp. 139-140.

6. - LUCIANO, Erodoto o Aezione, 4-6; per una diversa interpretazione: LOVISETTO, Luana, La pittura descritta: ekphrasis e riconversioni ecfrastiche nel Quattrocento e Cinquecento. Dans CENTANNI, Monica (éd.), L'originale assente. I'introduzione allo studio della tradizione classica. Milano : Mondadori, 2005, pp. 393-399. Oltre ai disegni di Raffaello all'Albertina di Vienna e al Tyelers Museum di Haarlem, l'esempio più celebre è l'affresco del Sodoma alla Villa Farnesina (1519), dove sulla sinistra compare un magnifico letto, molto simile a quello contemporaneo (1515) del Musée Lorrain, discusso da Pierre-Hippolyte Pénet. BARBIER, Muriel et PÉNET, Pierre-Hippolyte. « Le lit du duc Antoine de Lorraine et de la duchesse Renée de Bourbon: une nouvelle vie grâce au numérique ", In Situ [En ligne], 40|2019, mis en ligne le 15 septembre 2019, consulté le 30 septembre 2019. URL : http://journals.openedition.org/insitu/24089.

7. - LABORIE, Séverine. «'Mercure ordonne à Énée d'abandonner Didon' d'Orazio Samacchini: une oeuvre dévoilée par sa restauration ». La revue des musées de France, 2008, LVIII, 4, pp. 70-78.

8. - Inv. 1938 E 236, olio su tavola, cm $145 \times 110$. Riferita a un anonimo fiammingo, fu assegnata prudentemente a Calvaert da BREJON DE LAVERGNÉE, Arnaud, Dijon, Musée Magnin. Catalogue des tableaux et dessins italiens (XVe-XIX $X^{e}$ siècles). Paris : Réunion des Musées Nationaux, 1980, pp. 120-121, e con più convinzione da BENATI, Daniele, «Lorenzo Sabbatini: quadri 'con donne nude'». Dans BÉGUIN, Sylvie, DI GIAMPAOLO, Mario, Scritti di storia dell'arte in onore di Jürgen Winkelmann. Napoli : Paparo, 1999, p. 57 n. 22; sulla cronologia, vedi DANIELI, Michele, «Pittura erotica tra Bologna e Praga: aggiunte a Denys Calvaert e Dirck de Quade van Ravesteyn », Czech and Slovak Journal of Humanities, 2016, 3, pp.39-52; http://www2.culture.gouv.fr/public/mistral/ joconde_fr?

ACTION=RETROUVER\&FIELD_98=TOUT\&VALUE_98=calvaert\%20\&NUMBER=3\&GRP=0\&REQ=\%28\%28calvaert\%29\%20\%3aTOUT\%20\%29\& 9. - TITO LIVIO, Ab urbe condita, I, 58: "Vestigia viri alieni, Collatine, in lecto sunt tuo".

10. - L'attribuzione a Salviati era stata ribadita da GABRIELLI, Noemi, Galleria Sabauda. Maestri italiani. Torino : Ilte, 1971, p. 222, e ancora da MORTARI, Luisa, Francesco Salviati. Roma : De Luca, 1992, p. 121; sulla storia del dipinto vedi DANIELI, Michele, « Lorenzo Sabatini e Guido Reni. Per la provenienza di due dipinti della Galleria Sabauda », Studi Piemontesi, 2017, XLVI, 2, pp. 519-526.

11. - Lo scrittore bolognese Marcello Oretti la ricorda in palazzo Ratta a Bologna: BENATI, Daniele, «Lorenzo Sabbatini: quadri "con donne nude" ». Dans BÉGUIN Sylvie, DI GIAMPAOLO Mario, NARCISI Pietro (éd.), Scritti di storia dell'arte in onore di Jürgen Winkelmann. Napoli : Paparo, 1999, p. 51, ma la descrizione non corrisponde, e nel 17770 circa, quando Oretti scriveva, il quadro si trovava a Vienna già da mezzo secolo.

12. - Manca, dobbiamo ammetterlo, un esplicito riferimento al globo terrestre su cui la donna appoggia il piede.

13. - Il nome di Leonardo fu ripreso, tra gli altri, da LANZI, Luigi, Storia pittorica della Italia. Bassano : Remondini, 1795-1796, I, p. 107; il definitivo spostamento a Lavinia si deve a GHIRARDI, Angela, « Una pittrice bolognese nella Roma del Seicento: Lavinia Fontana », Il Carrobbio, 1984, X, 
pp. 158-161; CANTARO, Maria Teresa, Lavinia Fontana bolognese "pittora singolare" 1552-1614. Milano : Jandi Sapi, 1989, pp. 135-136; DERN, Alexandra, « Beobachtungen zu Kinderbildnissen in Euvre Lavinia Fontana». Dans DOMBROWSKI, Damian (éd.), Zwischen den Welten. Beiträge zur Kunstgeschichte für Jürg Meyer zur Capellen, Weimar : VGD, 2001, pp. 128-136; MURPHY, Caroline, Lavinia Fontana. A painter and her patrons in sixteenth-century Bologna. New Haven : Yale University Press, 2003, pp. 175-176; GRAZIANI, Irene, «Lavinia Fontana ». Dans BENTINI, Jadranka, CAMMAROTA Gian Piero, MAZZA, Angelo, SCAGLIETTI KELESCIAN, Daniela, STANZANI, Anna (éd.), Pinacoteca Nazionale di Bologna. Catalogo generale. 2. Da Raffaello ai Carracci, Venezia : Marsilo, 2006, pp. 209-210.

14. - MALVASIA, Carlo Cesare, Felsina pittrice. Bologna : Guidi all'Ancora, 1841, p. 178 n. 5.

\section{RIASSUNTI}

Alcuni esempi tratti dalla pittura Bolognese tra la fine del XVI e l'inizio del XVII secolo illustrano i vari significati che il letto può assumere nei diversi contesti narrativi. Il letto può diventare un grande palcoscenico dove Orazio Samacchini riscrive un famoso episodio dell'Eneide enfatizzandone i caratteri sensuali e privati. Le protagoniste femminili dei dipinti di Denys Calvaert vivono vicende tragiche, ma con differenti sfumature: la rettitudine morale di Lucrezia, la imprudenza di Semele impongono ambientazioni adeguate, e l'artista sceglie per ognuna il letto più adatto. Il letto che Lorenzo Sabatini inserisce in un suo dipinto invece ha un significato puramente simbolico, e il pittore se ne serve per arricchire una raffigurazione allegorica. La mancanza di una corte e di un luogo centrale di potere ha privato Bologna, durante l'età moderna, della produzione di manufatti e mobili destinati alla residenza di un sovrano. Il paradigma dell'arredamento di lusso è dunque da ricercare nella pittura manierista fiorentina $\mathrm{e}$ romana, o nelle stampe fiamminghe, piuttosto che in oggetti reali alla portata degli artisti. Lo dimostra in maniera evidente un dipinto da Lavinia Fontana, un vero "ritratto di letto": la sua esibizione di una agiatezza concreta lo rende molto diverso da quelli discussi in precedenza.

\section{INDICE}

Mots-clés : Letto, culla, Bologna, bolognese, cinquecento, seicento, pittura, iconografia, Orazio Samacchini, Daniele da Volterra, Denis Calvaert, Lorenzo Sabatini, Cesare Ripa, Lavinia Fontana, Iconologia

\section{AUTORE}

\section{MICHELE DANIELI}

Università di Bologna, cultore della materia micheledanieli@hotmail.com 